\title{
Phase Transitions in Brownian Pumps
}

\author{
Marcel Dierl ${ }^{1}$, Wolfgang Dieterich ${ }^{2}$, Mario Einax $^{1,3}$, and Philipp Maass ${ }^{1}$ \\ ${ }^{1}$ Fachbereich Physik, Universität Osnabrück, Barbarastraße 7, 49076 Osnabrück, Germany \\ ${ }^{2}$ Fachbereich Physik, Universität Konstanz, 78457 Konstanz, Germany \\ ${ }^{3}$ School of Chemistry, Tel Aviv University, Tel Aviv 69978, Israel
}

(Dated: 8 January 2013)

\begin{abstract}
We study stochastic particle transport between two reservoirs along a channel, where the particles are pumped against a bias by a traveling wave potential. It is shown that phase transitions of period-averaged densities or currents occur inside the channel when exclusion interactions between the particles are taken into account. These transitions reflect those known for the asymmetric simple exclusion process (ASEP). We argue that their occurrence is a generic feature of Brownian motors operating in open systems.
\end{abstract}

PACS numbers: 05.70.Ln, 05.40.-a, 05.60.-k

In connection with directed transport on the molecular level, two research areas have attracted great attention in the past: Brownian motors [1 6 ], and driven diffusion systems under a static bias [7-11]. Brownian motors are operated by a periodic process in time, where, in contrast to classical engines, fluctuations caused by thermal noise and thermally assisted overcoming of energy barriers are important [4, 12]. They exhibit some sort of symmetry breaking as a genuine feature [6], which is essential for their functioning. Driven diffusion under a static bias has received particular interest in connection with transport through open tube-like compartments because of boundary-induced phase transitions [13, 14]. Essential for their occurrence is the consideration of exclusion interactions, which means that two particles cannot occupy the same place.

We show in this work that boundary-induced phase transitions also appear in cyclically operating Brownian pumps, if exclusion interactions are taken into account. As argued further below, the occurrence of boundaryinduced phase transitions should be generic for Brownian motors with interacting particles in open environments. For demonstration we consider a model for a traveling wave potential [15], first introduced by Dhar and coworkers [16, 17].

The model is sketched in Fig. 11 A channel with $N$ sites connects two reservoirs $\mathrm{L}$ and $\mathrm{R}$ to the left and right with particle densities $\rho_{L}$ and $\rho_{R}$. Particles from these reservoirs are both injected into and ejected from the channel. The sites $i=1, \ldots, N$ can be occupied by at most one particle and a particle occupying site $i$ has energy $\varepsilon_{i}$. Likewise, a particle in the reservoirs $\mathrm{L}$ or $\mathrm{R}$ has energies $\varepsilon_{L}=\varepsilon_{0}$ or $\varepsilon_{R}=\varepsilon_{N+1}$. The particles inside the channel perform thermally activated jumps to vacant nearest-neighbor sites with rates $\Gamma_{i, i \pm 1}=\nu \exp \left[-\left(\varepsilon_{i \pm 1}-\varepsilon_{i}\right) / 2 k_{B} T\right]$, where $\nu$ is an attempt frequency and $k_{B} T$ the thermal energy. In the following, we choose $\nu^{-1}$ and $k_{B} T$ as our time and energy unit $\left(\nu=1, k_{B} T=1\right)$ and the site-to-site distance $a$ as length unit $(a=1)$. Along the channel, a constant bias
$F$ is present, leading to site energies linearly increasing from left to right. Accordingly, for $F>0$, a net particle flow from $\mathrm{R}$ to $\mathrm{L}$ occurs.

Application of a traveling wave potential from $\mathrm{L}$ to $\mathrm{R}$ can generate a particle flow against the bias. The potential modulates the site energies periodically in time and space with period $\tau$ and wavelength $\lambda$, yielding

$$
\varepsilon_{i}(t)=i F+A \sin \left(\frac{2 \pi i}{\lambda}-\frac{2 \pi t}{\tau}\right), \quad i=0, \ldots, N+1,
$$

where $A$ is the modulation amplitude. Because of the cyclic modulation of the reservoir energies and the site energies next to the reservoirs, the injection and ejection rates, irrespective of their detailed functional form, also vary periodically in time.

In the stationary state, the period-averaged net cur- (a)

(b)

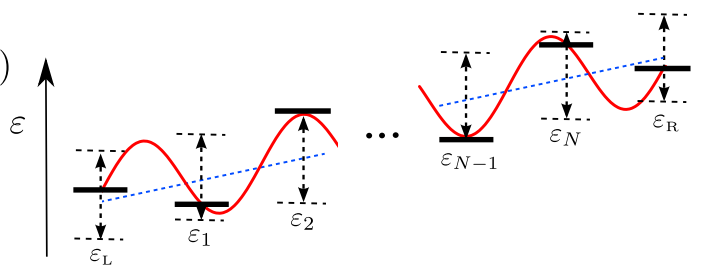

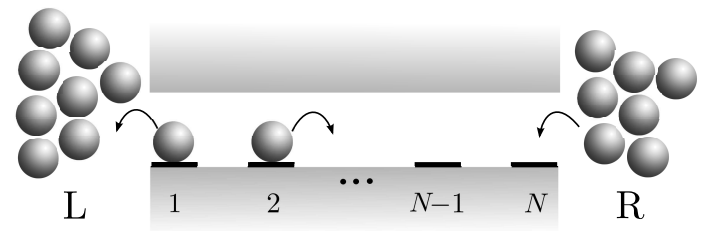

FIG. 1. (color online). (a) Sketch of a channel with $N$ equidistant sites in contact with two particle reservoirs $\mathrm{L}$ and $\mathrm{R}$ to the left and right. (b) Illustration of the site energies $\varepsilon_{i}$ along the channel. The dashed blue line marks a linear increase of the (time-averaged) site energies from left to right by a bias $F>0$. A time-dependent traveling wave potential allows for pumping of particles against the bias. Its superposition with the bias potential yields the total potential marked by the red solid line. 
rent $\bar{J}$ between two neighboring sites must be the same along the channel because of particle number conservation. Period-averaged particle densities $\bar{\rho}_{i}$ at sites $i$ are not spatially homogeneous. The form of the density profile depends on details of system-reservoir couplings specified by the injection and ejection rates. For the periodaveraged densities and currents we hence encounter a situation analogous to driven diffusion in an open system under static bias: Phase transitions of the bulk density $\bar{\rho}_{B}$ inside the channel should occur upon changing the densities $\rho_{L}$ and $\rho_{R}$ in the left and right reservoir.

An overview of the occurring phases is obtained based on the bulk current-density relation. This relation applies to channels with periodic boundary conditions, where the density $\bar{\rho}$ is homogeneous. In the following we refer to those as "closed channels". Figure 2(a) shows the bulk current-density relation for three different values of $F=0,1 / 2$, and 1 , and some fixed values of the other parameters. The results were obtained by kinetic Monte Carlo (KMC) simulations for time-dependent rates as described in [18]. For all $F$, there exists a density $\rho_{0}(F)$, where $\bar{J}$ changes sign. Current reversals have often been observed in Brownian motors, both in many-particle [16, 17, 19] and in one-particle models [3, 20, 21]. For $\bar{\rho}<\rho_{0}, \bar{J}$ goes through a maximum at $\bar{\rho}=\rho_{\max }$, and for $\bar{\rho}>\rho_{0}$ through a minimum at $\bar{\rho}=\rho_{\text {min }}$. The densities $\rho_{0}, \rho_{\max }$, and $\rho_{\min }$ all decrease with increasing $F$. In the absence of the bias, $F=0$, a sinusoidal behavior of $\bar{J}(\bar{\rho})$ is found, and $\bar{J}(\bar{\rho})=-\bar{J}(1-\bar{\rho})$. This property is a consequence of particle-hole symmetry, and the sinusoidal form can be understood from a perturbative treatment in the wave amplitude $A[16,17]$.

Let us now consider the open channel in Fig. 1. In this open channel the particle number is fluctuating in the stationary state and density profiles $\bar{\rho}_{i}$ form with a flat regime $\bar{\rho}_{i}=\bar{\rho}_{B}$ inside the channel (see inset of Fig. 3 for examples). The density $\bar{\rho}_{B}$ and, accordingly, the appearance of the different phases depends on the type of system-reservoir couplings [22, 23]. Their identification in general requires the scanning of all possible values of $\rho_{L}$ and $\rho_{R}$ for the open channel. However, for a particular choice of coupling, coined "bulk-adapted", $\bar{\rho}_{B}$ can be derived from the curves in Fig. 2(a) by applying minimum/maximum current principles. These were first introduced for diffusion systems under static bias $[13,24,25]$ and it is rather surprising at first sight that they can be taken over to time-varying driving forces. The reason is that, as under static bias, a continuity equation relates the period-averaged densities and currents, with a source-free $\bar{J}$ in the stationary regime. Applied to the period-averaged quantities, the principles state that if $\rho_{L}$ is smaller (larger) than $\rho_{R}$, the bulk density $\bar{\rho}_{B}$ equals the density, where $\bar{J}(\bar{\rho})$ assumes its minimal (maximal) value in the intervals $\rho_{L}<\bar{\rho}<\rho_{R}$ $\left(\rho_{R}<\bar{\rho}<\rho_{L}\right)$. This is a consequence of the fact that, to match the reservoir densities at the boundaries, the (a)

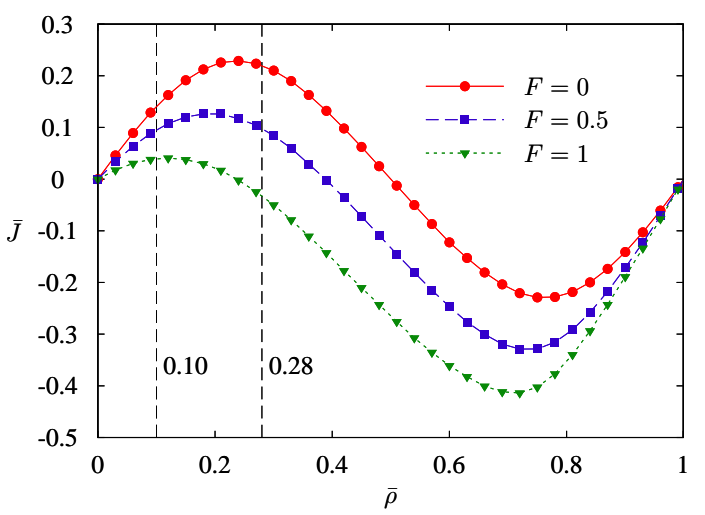

(b)

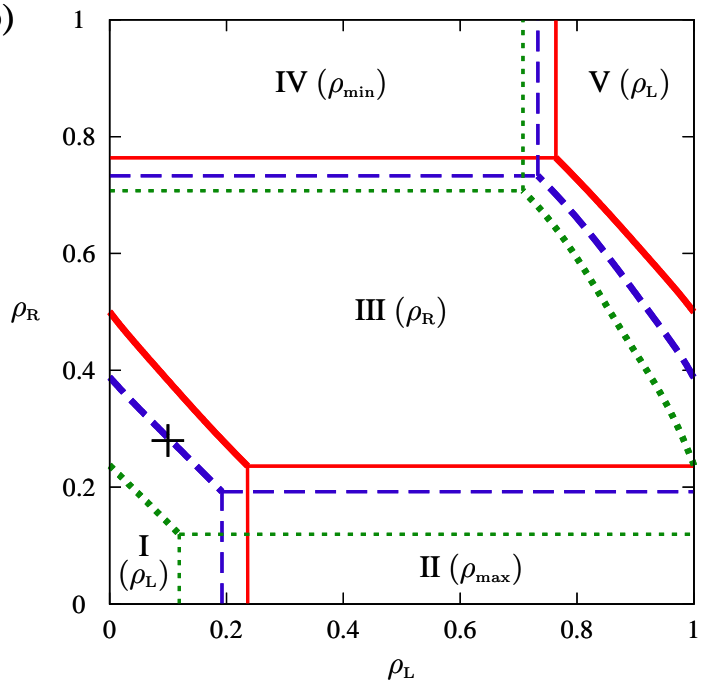

FIG. 2. (color online). (a) Period-averaged current $\bar{J}$ as a function of period-averaged density $\bar{\rho}$ in a bulk system for three different $F$ values, and fixed parameters $\lambda=4, \tau=2$, and $A=3$ of the traveling wave potential. Data were obtained by KMC simulations of a closed channel. (b) Phase diagram derived from (a) by applying the minimum/maximum current principles. Phase transitions of first order are marked by thick lines, and transitions of second order by thin lines. The assignment of line types (and colors) to the $F$ values is the same as in the legend of (a). The five different phases are labeled by roman numbers with respective bulk densities given in brackets. The cross marks the point $\left(\rho_{L}, \rho_{R}\right)=(0.1,0.28)$ used in the example for demonstrating the influence of a phase transition on $\bar{J}$ in Fig. 3 in (a) the densities 0.1 and 0.28 are marked by vertical dashed lines.

period-averaged density profiles cannot be uniform, and accordingly diffusive currents occur. These diffusive currents plus $\bar{J}(\bar{\rho})$ must be constant everywhere. For example, if, for $\rho_{L}<\rho_{R}, \bar{\rho}_{i}$ increases monotonically from left to right, the diffusive current is negative, or zero for $\bar{\rho}_{i}=\bar{\rho}_{B}$. Accordingly, to keep the sum of $\bar{J}(\bar{\rho})$ and the diffusive current constant, $\bar{J}(\bar{\rho})$ must be minimal in the region of flat density $\bar{\rho}_{B}$. The technical details for implementing the bulk-adapted couplings are described in 26.

Applying the minimum/maximum current principles 
to the curves in Fig. 2 (a) yields the five phases shown in Fig. 2(b), where $\bar{\rho}_{B}$ equals either $\rho_{L}$ (phases I, V), $\rho_{R}$ (phase III), $\rho_{\max }$ (phase II) or $\rho_{\min }$ (phase IV). Firstorder transitions between two phases with jumps of $\bar{\rho}_{B}$ are marked by thick lines, and second-order transitions are marked by thin lines. These transition lines shift when $F$ changes. In phases I and $\mathrm{V}$ the density profiles are flat on the left side (cp. the profiles for $F=0.2$ and 0.4 in the inset of Fig. (3), in phase III on the right side (cp. the profiles for $F=0.6$ and 0.8 in the inset of Fig. 3), and in the maximum and minimum current phases II and IV, the density profile is flat in the channel's interior and bent towards $\rho_{L}$ and $\rho_{R}$ at the two boundaries.

The phase transitions influence the current $\bar{J}$ and hence the efficiency of the Brownian pump. To demonstrate this, let us consider, as an example, the particle flow against increasing bias $F$, for fixed reservoir densities $\rho_{L}=0.1$ and $\rho_{R}=0.28$, and the same parameters of the traveling potential as in Fig. 2. The corresponding point $\left(\rho_{L}, \rho_{R}\right)=(0.1,0.28)$ is marked by the cross in Fig. 2(b). Starting from $F=0$, this point lies in phase I $\left(\bar{\rho}_{B}=0.1\right)$, and at large $F=1$ it lies in phase III $\left(\bar{\rho}_{B}=0.28\right)$. The transition occurs at $F=F_{\star}$ with $F_{\star} \simeq 0.5$. Density profiles from KMC simulations before $\left(F<F_{\star}\right)$ and after this transition $\left(F>F_{\star}\right)$ are shown in the inset of Fig. 3. At $\bar{\rho}=0.1$, the current $\bar{J}$ in Fig. 2(a) is more weakly varying with $F$ than at $\bar{\rho}=0.28$. Accordingly, $\bar{J}$ as a function of $F$ displays a kink at the transition point $F=F_{\star}$, see Fig. 3. The open symbols in the figure are from KMC simulations of closed channels with $\bar{\rho}=0.1$ for $F<F_{\star}$ and $\bar{\rho}=0.28$ for $F>F_{\star}$. The four filled symbols are the currents in the open channels with the density profiles shown in the inset. The agreement of $\bar{J}$ for the open and closed channel confirms the validity of the minimum/maximum current principles.

The effect of the shifting of phase transition lines on $\bar{J}$ has been demonstrated here for a change of the bias. A shifting of transition lines with corresponding effects on $\bar{J}$ can also be induced by a variation of the parameters of the traveling potential. This is relevant for the efficiency and optimization of the pump. The occurrence of minimum and maximum current phases has an interesting implication for the robustness of the motor's performance against changes of the reservoir densities, because in these phases $\bar{\rho}_{B}$ and hence $\bar{J}$ are independent of $\rho_{L}$ and $\rho_{R}$ [27].

For Brownian motors of other type, as, for example, a flashing or rocking ratchet, analogous features are to be expected, if they operate in an open environment. This is because, after period-averaging of currents and densities, a net driving force (for the motor to transport particles) and local particle number conservation (leading to a coupling between currents and densities) are present. Phase transitions will appear also for Brownian motors treated in continuum, if particles with some hard-sphere diameter are considered. In fact, the model in Fig. 1 can

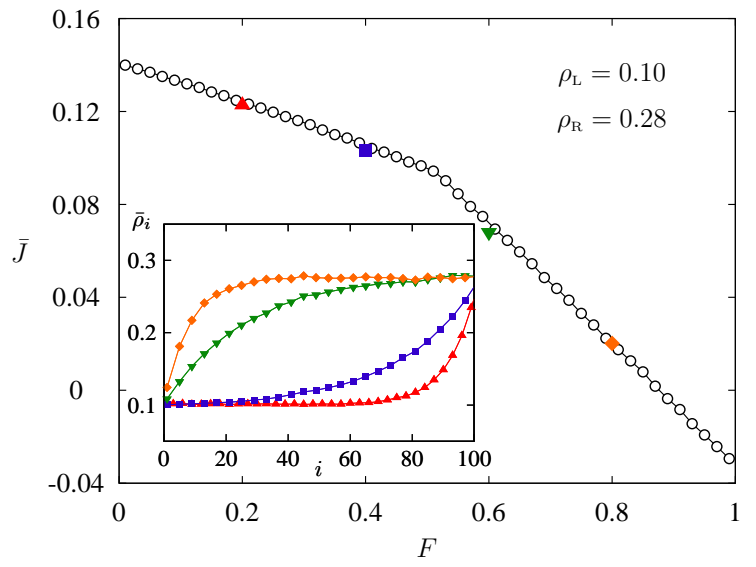

FIG. 3. (color online). Current $\bar{J}$ as function of $F$ for fixed reservoir densities and the same parameters of the traveling wave potential as in Fig. 2. The open circles are for closed channels. The filled colored symbols refer to $\bar{J}$ in open channels at $F=0.2,0.4,0.6$, and 0.8 , for which the respective density profiles are shown in the inset.

be easily connected to a discrete implementation of a Langevin equation [16]. For particle interactions beyond site exclusion, more complex phase diagrams can occur 22, 25]. Likewise, richer phase diagrams can emerge by injection and ejection processes along filaments according to a Langmuir kinetics [28].

[1] F. Jülicher, A. Ajdari, and J. Prost, Rev. Mod. Phys. 69, 1269 (1997).

[2] R. D. Astumian, Science 276, 917 (1997).

[3] P. Reimann, Phys. Rep. 361, 57 (2002).

[4] R. D. Astumian and P. Hänggi, Phys. Today 55 (11), 33 (2002).

[5] E. R. Kay, D. A. Leigh, and F. Zerbetto, Angew. Chem. Int. Ed. 46, 72 (2007).

[6] P. Hänggi and F. Marchesoni, Rev. Mod. Phys. 81, 387 (2009).

[7] B. Derrida, Phys. Rep. 301, 65 (1998).

[8] G. M. Schütz, in Phase Transitions and Critical Phenomena, Vol. 19, edited by C. Domb and J. L. Lebowitz (Academic Press, San Diego, 2001).

[9] O. Golinelli and K. Mallick, J. Phys. A 39, 12679 (2006).

[10] R. A. Blythe and M. R. Evans, J. Phys. A 40, R333 (2007).

[11] A. B. Kolomeisky, J. Phys.: Condens. Matter 25, 463101 (2013).

[12] M. O. Magnasco, Phys. Rev. Lett. 71, 1477 (1993).

[13] J. Krug, Phys. Rev. Lett. 67, 1882 (1991).

[14] S. Klumpp and R. Lipowsky, J. Stat. Phys. 113, 233 (2003).

[15] Sometimes the term 'Brownian motors' is reserved for those motors, where thermal noise facilitates directed particle motion. The traveling wave potential is not of that sort, but the general concepts presented in this work equally apply to them. 
[16] D. Chaudhuri and A. Dhar, Europhys. Lett. 94, 30006 (2011).

[17] K. Jain, R. Marathe, A. Chaudhuri, and A. Dhar, Phys. Rev. Lett. 99, 190601 (2007).

[18] V. Holubec, P. Chvosta, M. Einax, and P. Maass, Europhys. Lett. 93, 40003 (2011).

[19] I. Derényi and T. Vicsek, Phys. Rev. Lett. 75, 374 (1995).

[20] P. Reimann and P. Hänggi, Appl. Phys. A 75, 169 (2002).

[21] M. Kostur and J. Łuczka, Phys. Rev. E 63, 021101 (2001).

[22] M. Dierl, P. Maass, and M. Einax, Phys. Rev. Lett. 108, 060603 (2012).

[23] M. Dierl, M. Einax, and P. Maass, Phys. Rev. E 87, 062126 (2013).
[24] V. Popkov and G. M. Schütz, Europhys. Lett. 48, 257 (1999).

[25] J. S. Hager, J. Krug, V. Popkov, and G. M. Schütz, Phys. Rev. E 63, 056110 (2001).

[26] See supplemental material at http://[.].

[27] For transitions into and out of the minimum and maximum current phases induced by one (or several) varying control parameters, the currents before and after the transition have a different functional dependence on these parameters. The functional forms cross with continuous first-order derivative, but there will be singularities in higher-order derivatives.

[28] A. Parmeggiani, T. Franosch, and E. Frey, Phys. Rev. Lett. 90, 086601 (2003). 